\title{
Malignant Adult Hemangiopericytoma
}

National Cancer Institute

\section{Source}

National Cancer Institute. Malignant Adult Hemangiopericytoma. NCI Thesaurus. Code C7946.

A malignant hemangiopericytoma occurring in the adult population. 